Proc. of the 13th Symposium of Magnetic Measurements and Modeling SMMM'2018, Wieliczka, Poland, October 8-10, 2018

\title{
Verification of Bertotti's Loss Model for Non-Standard Excitation
}

\author{
B. KOPRIVICA ${ }^{a, *}$ AND K. CHWASTEK ${ }^{b}$ \\ ${ }^{a}$ University of Kragujevac, Faculty of Technical Sciences in Cacak, Svetog Save 65, 32000 Cacak, Serbia \\ ${ }^{b}$ Częstochowa University of Technology, Faculty of Electrical Engineering, \\ al. Armii Krajowej 17, 42-200 Częstochowa, Poland
}

\begin{abstract}
This paper focuses on the possibility to use the Bertotti loss theory to describe energy losses in a toroidal core made of grain-oriented steel sheet under non-standard excitation conditions. The temporal $H_{\text {clas }}(t)$ and $H_{\text {exc }}(t)$ dependences are computed using a simple numerical scheme, which may be easily implemented in a spreadsheet.
\end{abstract}

DOI: 10.12693/APhysPolA.136.709

PACS/topics: 75.20.En, 75.60.-d, 75.60.Ej, 85.70.Ay

\section{Introduction}

Grain-oriented electrical steels are an important group of soft magnetic materials (SMMs), indispensable for the design of contemporary transformers [1-3]. Although the technology of their production has been improved significantly in recent years, it is estimated that losses in magnetic circuits of electrical machines still account for $5-10 \%$ of total electrical energy produced worldwide [2], thus the research on properties of grain-oriented steels is crucial in the era of depleted fossil fuels. A better understanding of energy dissipation phenomena may result in an improvement of efficiency of electrical machines. In European Union this issue has been the subject of concern of European Commission (Regulation No. 640/2009 of 22.07.2009).

There are many approaches to the description of losses in grain-oriented electrical steels [4]. One of the most important models was advanced by Pry and Bean [5], who considered the issue of loss prediction for a periodic structure consisting of $180^{\circ}$ domain walls. The authors calculated the energy loss due to eddy currents for different ratios $2 L / d$, where $2 L$ denotes average domain wall spacing in demagnetized state and $d$ denotes sheet thickness. Their results were useful for drawing some qualitative conclusions on the effect of domain wall structure on energy losses in ferromagnetic materials. Spatial inhomogeneity of magnetic permeability, being an unavoidable consequence of the existence of domain structure, must lead to increased losses due to the flow of eddy currents. In the Pry-Bean model it was assumed that all domain walls moved with the same velocity and the number of active domain walls remained constant for any excitation frequency. These assumptions were later criticized by Bertotti [6] as oversimplified and

\footnotetext{
* corresponding author; e-mail: branko.koprivica@ftn.kg.ac.rs
}

not reflecting real conditions. His formalism, developed in the eighties of the last century $[7,8]$, is the subject of the present paper.

It should be remarked that the formulae developed by Bertotti are generally valid for sine induction waveform. In reality, the excitation conditions may differ significantly from those prescribed in international standards. Therefore, it is crucial to examine the possibility to use the formalism for generic $B$-waveforms.

In the present paper we examine the possibility to use the Bertotti formulae for classical and excess loss computation for triangular $H$-waveforms in a toroidal core made of grain-oriented steel. Such excitation produces nonsinusoidal $B$-waveforms under constant rate of change of magnetising field $(\mathrm{d} H(t) / \mathrm{d} t=$ const), which is more complicated case for the analysis than the usual case when $B$ waveform is sinusoidal.

\section{The Bertotti model}

The approach advanced by Bertotti relies on implicit decomposition of total loss into three terms $P_{\text {total }}=$ $P_{\text {hyst }}+P_{\text {clas }}+P_{\text {exc }}$. The first term is related to the area of hysteresis loop under quasi-static excitation, the second one is obtained from the solution of Maxwell's equations and describes losses due to eddy currents in the bulk material. The third term is the so-called excess or anomalous loss, its presence is attributed by the model developer to eddy currents flowing around moving domain walls within the sample.

In the Bertotti theory it is assumed that the effect of local interactions between neighboring domain walls may be described with the concept of "magnetic object" (MO). An abstract $\mathrm{MO}$ may be interpreted as a single domain wall in a crystal in which there are few $180^{\circ}$ Bloch walls, and in that case dynamic interactions between different walls are neglected [8]. In materials with fine grain structure, where spatial fluctuations of coercive field strength are controlled by grain size, MOs are identified as grains. Macroscopic description of magnetization changes may 
be defined by the determination of dynamics of statistically independent MOs. Thus, loss estimation issue reduces to the examination of dependences of $n$ MOs on frequency $f$, maximum induction $B_{\mathrm{m}}$, and microstructure. The external magnetic field with uniform spatial distribution tends to introduce a uniform distribution of magnetization in the sample cross-section. In a material with homogeneous structure the state of uniform magnetization is indeed obtained and the losses due to magnetization process are given with the expression for classical loss $P_{\text {clas }}$.

The structural non-homogeneities of the material, acting as sources of internal fields with highly non-uniform spatial distribution, hamper the tendency to obtain a uniform magnetization distribution.

Assuming that $H_{\text {exc }}(t)$ is the field strength supplied from the source necessary to compensate the field strengths from eddy currents generated by MOs, the average value of excess loss may be written as

$$
P_{\text {exc }}=\frac{1}{T} \int_{0}^{T} H_{\text {exc }}(t) \frac{\mathrm{d} B(t)}{\mathrm{d} t} \mathrm{~d} t .
$$

Also, it can be assumed that the excess field strength is proportional to the rate of magnetic flux induced by moving MOs, and one can write

$$
H_{\mathrm{exc}}(t)=G \sigma\left\langle\frac{\mathrm{d} \Phi(t)}{\mathrm{d} t}\right\rangle,
$$

where the constant $G \cong 0.1356$ and $\sigma$ is material conductivity. The rate of total flux depends on $n(t)$ according to

$$
S \frac{\mathrm{d} B(t)}{\mathrm{d} t}=n(t)\left\langle\frac{\mathrm{d} \Phi(t)}{\mathrm{d} t}\right\rangle,
$$

where $S$ is lamination cross-section area. Therefore, the following relationship may be written:

$$
H_{\text {exc }}(t)=\sigma G S \frac{1}{n(t)} \frac{\mathrm{d} B(t)}{\mathrm{d} t} .
$$

On the other hand, the number of active MOs depends on $H_{\text {exc }}(t)$. This dependence may be written for grainoriented steel subject to magnetization along the rolling direction as

$$
n(t) \cong n_{0}+\frac{H_{\mathrm{exc}}(t)}{V_{0}},
$$

where $n_{0}$ and $V_{0}$ are parameters related to the material microstructure [8]. Parameter $n_{0}$ is the number of active MOs when the frequency tends to zero and $V_{0}$ is the magnetic field that shows the ability of the external magnetic field to increase the number of active MOs with the increase of the frequency. The two last expressions may be combined yielding an implicit relationship for $H_{\text {exc }}(t)[9,10]$, obtained as the positive root of the quadratic equation, which is to be fulfilled at any time instant

$$
\frac{1}{V_{0}} H_{\mathrm{exc}}^{2}+n_{0} H_{\mathrm{exc}}-\sigma G S \frac{\mathrm{d} B(t)}{\mathrm{d} t}=0
$$

$$
H_{\text {exc }}=\frac{-n_{0}+\sqrt{n_{0}^{2}+\left(4 \sigma G S / V_{0}\right) \mathrm{d} B / \mathrm{d} t}}{2 / V_{0}} .
$$

It is clear that the relationship $H_{\text {exc }}(t)$, as well as the one for $P_{\text {exc }}(t)$, depends on the shape of $\mathrm{d} B / \mathrm{d} t$ waveform. In magnetic measurements carried out in accordance with international standards the cosine $\mathrm{d} B / \mathrm{d} t$ waveform is maintained, but in practical applications it may be different. It should be noted that Eq. (2) holds for non-negative $\mathrm{d} B(t) / \mathrm{d} t$. Its absolute value taken with minus sign should be considered for time instants when takes negative values, as it can be deduced from the literature $[8,11]$.

\section{Modelling}

In order to verify whether Bertotti's theory holds for non-standard excitations, several measurements were carried out on a toroidal sample made of grain-oriented electrical steel (grade 27PH100 from POSCO) using a LabVIEW application, which made it possible to control the $H$ and $B$ waveforms. Strip thickness and width are $0.27 \mathrm{~mm}$ and $15 \mathrm{~mm}$, respectively. The inner radius of the sample is $45 \mathrm{~mm}$ and the outer radius is $52.5 \mathrm{~mm}$. The mass of the sample is $0.241 \mathrm{~kg}$. The measurement setup consisted of programmable power supply, data acquisition card NI 9205 and PC with a data acquisition application made in LabVIEW software. More details may be found in $[12,13]$.

The values of model parameters $n_{0}$ and $V_{0}$ were determined from $P=P(f)$ measurements carried out under standard excitation conditions (sine $B(t)$ waveform). Measurements were performed under dynamic and quasi-static regime and excess losses were calculated. Also, according to the average approach given in [8], $H_{\text {exc }}$ and $n$ were calculated for different values of $B$. Dependence of $n$ on $H_{\text {exc }}$ is represented as linear function with parameters $n_{0}$ and $V_{0}$, as it has been given in the previous section. The estimated values were $n_{0}=26$ and $V_{0}=0.3278 \mathrm{~A} / \mathrm{m}$. These values were kept constant during subsequent modelling for non-standard excitation conditions, because the original theory did not account their possible dependence on induction level. Yet it should be remarked that Kowal et al. [11] consider some possible variations of these values. The estimated values are comparable to those used by Bertotti in his papers. The value of $P_{\text {hyst }}$ was computed from the Steinmetz equation and was assumed to be independent of the $B(t)$ waveform shape.

Figure 1 depicts $\mathrm{d} B(t) / \mathrm{d} t$ obtained from the measured induced voltage and reconstructed $B(t)$ dependences for triangular high-level $H(t)$ excitation at $f=50 \mathrm{~Hz}$. Figure 2 depicts the computed $H_{\text {clas }}(t)$ and $H_{\text {exc }}(t)$ dependences over one period. Their waveforms follow spiky shape of $\mathrm{d} B / \mathrm{d} t$. It can be noticed that, outside that spike, $H_{\text {clas }}$ has very low level, while $H_{\text {exc }}$ has a significantly higher value. Maximum of $H_{\text {exc }}$ is about $30 \%$ higher than maximum of $H_{\text {clas }}$. 


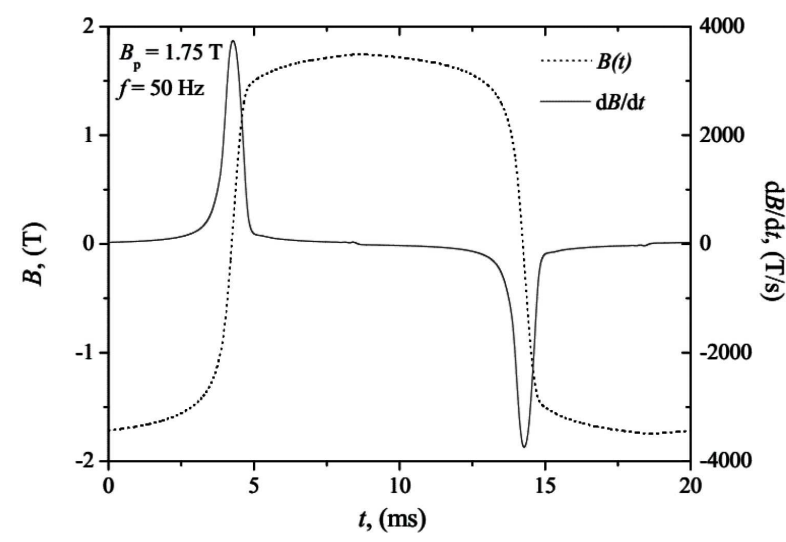

Fig. 1. Dependences of $\mathrm{d} B(t) / \mathrm{d} t$ and $B(t)$ for triangular high-level $H(t)$ field.

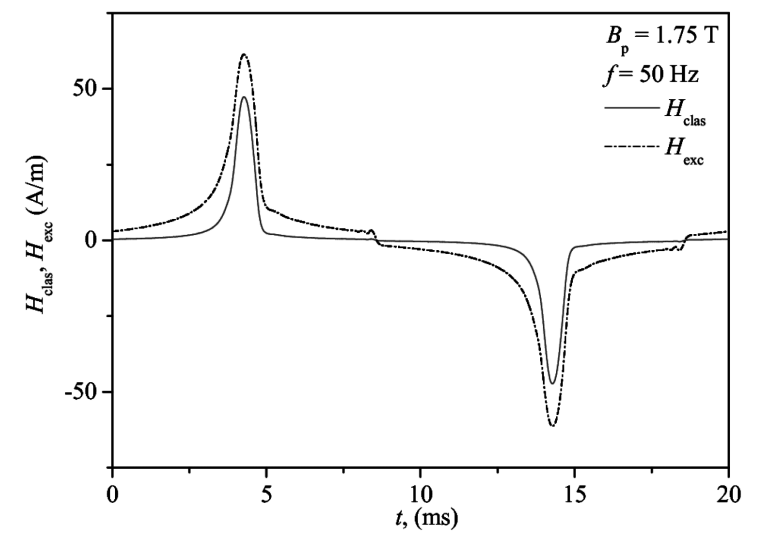

Fig. 2. Computed $H_{\text {clas }}(t)$ and $H_{\text {exc }}(t)$ dependences.

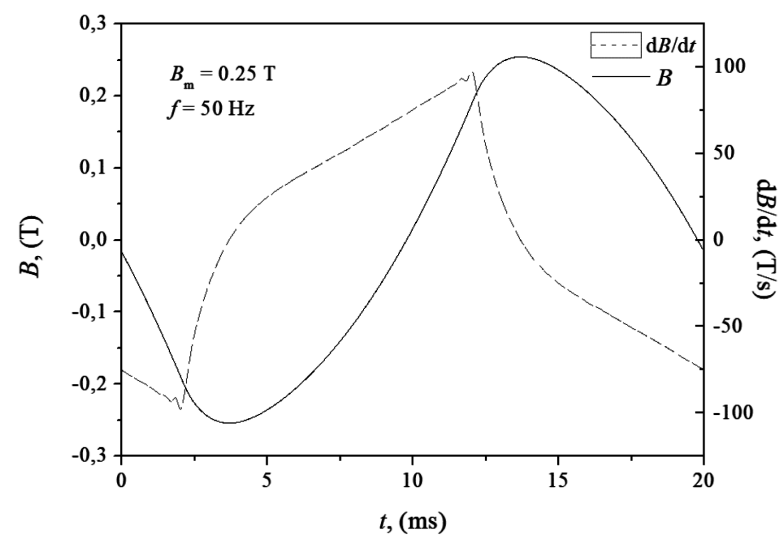

Fig. 3. Dependences of $\mathrm{d} B(t) / \mathrm{d} t$ and $B(t)$ for triangular low-level $H(t)$ field.

After time averaging (integration over the period) of the computed dependences and summing all loss components together we were able to estimate total losses dissipated in the sample. The result was compared to the measured value of total losses. It was found that in the considered case the value from computations exceeded the measured one by $2.1 \%$.

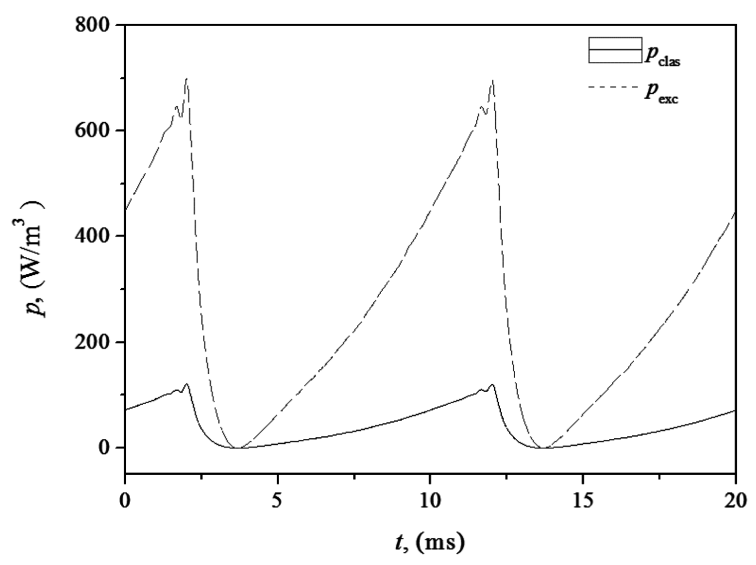

Fig. 4. Computed $p_{\text {clas }}(t)$ and $p_{\text {exc }}(t)$ dependences for triangular low-level $H(t)$ field.

Figures 3 and 4 depict a case of triangular low-level $H(t)$ excitation. The induction amplitude was $0.25 \mathrm{~T}$ and the excitation frequency was $50 \mathrm{~Hz}$. Figure 3 depicts $\mathrm{d} B(t) / \mathrm{d} t$ and $B(t)$ dependences, whereas in Fig. 4 the instant values of loss components are shown. In this case the averaged value of total loss from calculations was about $39.4 \%$ larger than its experimental counterpart.

\section{Conclusions}

In this paper we have considered the problem whether Bertotti's three term loss formula might be applied for non-standard excitation cases. We have focused on triangular $H(t)$ excitation applied to a toroid sample made of grain-oriented steel.

We have found out that it is possible to reconstruct temporal $H_{\text {clas }}(t)$ and $H_{\text {exc }}(t)$ dependences and to estimate the values of total losses using a numerical approximation of the derivative $\mathrm{d} B(t) / \mathrm{d} t$. The proposed approach may be easily implemented in a spreadsheet.

The maximum error for the considered cases was around $39 \%$. The discrepancies between the modeled and the measured loss values may be attributed to the simplifying assumptions (assumption of constant values for $n_{0}$ and $V_{0}$ ) as well to different mechanisms governing the magnetization dynamics at different parts of the hysteresis curve.

Future work shall be focused on fine tuning of the description for lower excitation levels, because in this case we have noticed considerable discrepancies between the measured and the modeled loss values. We suspect that this effect may be due to a change in monotonicity of the $P_{\text {tot }}(f)$ curve (a crossover phenomenon) in the quasistatic regime, noticed and described for the first time by De Wulf et al. [14]. Moreover, we plan to test the approach for a wider class of excitation signals and for materials with more complicated structures (microcrystalline $6.5 \% \mathrm{Si}$ steel, amorphous and nanocrystalline alloys). 


\section{References}

[1] S.V. Kulkarni, S.A. Khaparde, Transformer Engineering: Design, Technology, and Diagnostics, CRC Press (Taylor \& Francis Group), London 2012.

[2] A.J. Moses, Scr. Mater. 67, 5 (2012).

[3] A. Krings, J. Soulard, J. Electr. Eng. 10, 3 (2010).

[4] K. Chwastek, A.P.S. Baghel, P. Borowik, B.S. Ram, S.V. Kulkarni, in: 2016 Progress in Applied Electrical Engineering, Koscielisko-Zakopane (Poland), IEEE, 2016.

[5] R.H. Pry, C.P. Bean, J. Appl. Phys. 29, 532 (1958).

[6] G. Bertotti, J. Magn. Magn. Mater. 54, 3 (1986).

[7] G. Bertotti, IEEE Trans. Magn. 24, 1 (1988).

[8] G. Bertotti, Hysteresis in Magnetism, Academic Press, San Diego 1998.
[9] J. Szczyglowski, Modern Soft Magnetic Materials - Chosen Properties, Wyd. Politechniki Częstochowskiej, Częstochowa 2013 (in Polish).

[10] J. Szczyglowski, J. Electr. Eng. 69, 6 (2018).

[11] D. Kowal, P. Sergeant, L. Dupré, L. Vandenbossche, IEEE Trans. Magn. 51, 1 (2015).

[12] B. Koprivica, A. Milovanović, M. Djekić, Serb. J. Electr. Eng. 6, 3 (2009).

[13] B. Koprivica, I. Dumitru, A. Milovanović, O. Caltun, Facta Universitatis Series Electron. Energet. 30, 1 (2017).

[14] M.D. Wulf, L. Dupré, J. Melkebeek, J. Appl. Phys. 87, 9 (2000). 